\title{
FREQUENCIES OF A HORIZONTALLY SUSPENDED COILED SPRING.
}

By F. J. Rogers.

A LONG coiled spring of large section suspended by cords so A that its axis is horizontal is often used to illustrate longitudinal wave-motion. As far as I know this form of apparatus was first described by Weinhold. ${ }^{1}$ I have constructed several such "wave-machines" and have found them very effective in teaching the phenomena of longitudinal wave-motion. With the aid of such a machine one can illustrate, advancing waves of compression and of rarefaction; reflection of waves both with and without change of sign ; standing waves, including all the modes of vibration of the air in open and closed organ-tubes. With the last machine that I constructed it is easy to obtain standing waves for all the frequencies of which the spring is capable up to and including the tenth mode, counting the fundamental as the first. The frequency of the highest of these modes is less than four, so that these frequencies can be determined numerically merely by counting the vibrations. For a really successful wave-machine of this kind the cross-section of the coil must be great as compared with the diameter of the wire used. In case of the one above mentioned the wire used was No. I8; there were 300 spires, each being $5 \mathrm{~cm}$. in diameter. The length of the spring was $2.4 \mathrm{~m}$.; it was suspended by 25 pairs of threads, each pair consisted of two threads forming an angle of about $35^{\circ}$, each being $60 \mathrm{~cm}$. long.

In using such a wave-machine I have often been reminded of the fact that the wave-motion dealt with is not wholly due to elasticity. It is obvious that the force of gravity must operate to affect the motion, more or less, on account of the suspension being pendulum fashion. Just what this gravity effect may be, is not at all obvious. Upon first thought one might say, that as the gravity

${ }^{1}$ Physikalische Demonstrationen, Zweite auflage, p. 206. 
return force is proportional to displacement (when displacements are small) the effect would be the same as if the spring were stiffer, giving as a result an increase of all frequencies in the same ratio. That this is not true is readily proved by determining the frequencies of several modes of vibration. These frequencies obviously do not belong to a harmonic series as they would if the vibrations were wholly due to elasticity or as they would if all frequencies were increased in the same ratio by the gravity effect.

The wave-lengths of the different modes of vibration are, however, the same as would be the case if the vibrations were wholly due to elasticity. This is proved by the fact that the nodes for all modes of vibration are located just where they would be in the case of purely elastic vibrations. In the case of a coiled spring, whether suspended horizontally or not, the nodes are located with respect to the number of spires, and not with reference to length

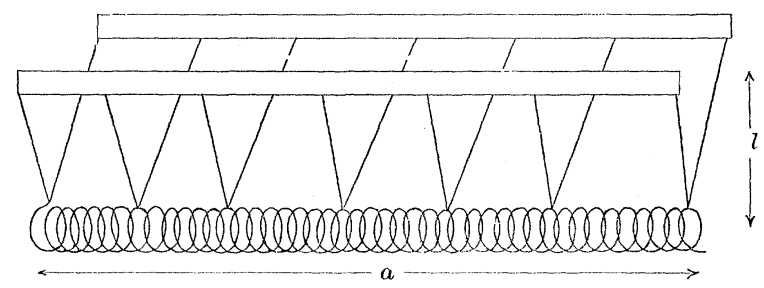

Fig. 1.

simply; for example, a coiled spring free at both ends and vibrating in its fundamental mode will have a node at the middle spire or middle point of the wire. If the spires are not equidistant this may be nearer one end of the spring than the other.

Assuming nodes to be located as in the case of elastic vibrations, it is easy, by the energy method, to compute any or all the frequencies of a horizontally suspended spring. We shall also assume that the motion of any part of the spring is truly simply harmonic. A more general assumption would merely make the mathematical work longer without any advantage, as we are not at present interested in the relative amplitude of the components for any particular method of starting.

Let length of coil be $a$, length of suspending cords be $l$, mass of unit length of spring be $m$, and force required to double the length of the spring be $F$. See Fig. I. 
Consider the motion of a portion of the spring from a node to the next anti-node and let this length be $\alpha / s$. If the spring is "free" at both ends or "clamped" at both ends, $s$ may have any of the values, $2,4,6,8, \ldots$ If the spring is free at one end and clamped at the other end $s$ may have any of the values, I, 3, 5, 7, . . Let the origin be at a node and $x$ be the distance of a spire of the spring from the origin while $y$ is the displacement of this spire from its normal or equilibrium position. Finally, let $y_{a}$ be the displacement of the spire at the anti-node. These quantities are represented in Fig. 2. Displacements are of course longitudinal but they are represented in the upper part of the figure as though they were transverse.

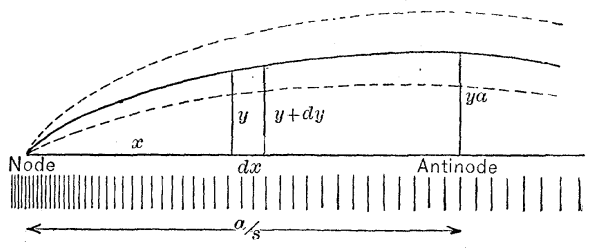

Fig. 2.

According to the assumption already made the velocity of any point of the spring is a simple harmonic function of $x$, the distance of that point from the origin;

$$
\therefore \dot{y}=\dot{y}_{a} \sin \frac{s \pi x}{2 a} \text {. }
$$

The kinetic energy of the spring is given by the following integral :

$$
T=\frac{m}{2} \int \dot{y}^{2} d x
$$

taken between the proper limits. In the present case this becomes for the whole spring

$$
T=\frac{s m \dot{y}_{a}^{2}}{2} \int_{0}^{a / s} \sin ^{2} \frac{s \pi x}{2 a} d x=\frac{m a \dot{y}_{a}^{2}}{4} .
$$

The potential energy of the spring depends upon its elastic distortion and also upon the distance its separate spires are lifted, against gravity, above their normal position.

Consider any portion of the spring of length $d x$. When dis- 
torted its length is $d x+d y$ and the force required to maintain this distortion is $F \frac{d y}{d x}$. The work required to produce this distortion is half the final force times the displacement or

$$
\frac{1}{2} F \frac{d y}{d x} d y=\frac{1}{2} F \frac{d y}{d x} \frac{d y}{d x} d x
$$

The elastic potential energy is therefore

$$
U^{\prime}=\frac{1}{2} F \int\left(\frac{d y}{d x}\right)^{2} d x .
$$

According to the original assumption the displacement of any spire is a simple harmonic function of its distance from the origin or

$$
y=y_{a} \sin \frac{s \pi x}{2 a} .
$$

Substituting this value of $y$ in (5) and integrating we obtain, for the whole spring,

$$
U^{\prime}=s \cdot \frac{F}{2} \cdot \frac{s^{2} \pi^{2}}{4 a^{2}} y_{a}^{2} \int_{0}^{a / s} \cos ^{2} \frac{s \pi x}{2 a} d x=\frac{s^{2} \pi^{2} F}{\mathrm{I} 6 a} y_{a}^{2} .
$$

The gravitational potential energy of any element of the spring of length $d x$ and mass $m d x$ is the same as the potential energy of a simple pendulum whose bob has the same mass and is displaced horizontally a distance represented by $y$. This potential energy is therefore

$$
U^{\prime \prime}=\frac{m g}{2 l} \int y^{2} d x
$$

substituting for $y$ from (6), integrating between the proper limits, we have for the whole spring

$$
U^{\prime \prime}=s \frac{m g y_{a}^{2}}{2 l} \int_{0}^{a / s} \sin ^{2} \frac{s \pi x}{2 a} d x=\frac{m g a y_{a}^{2}}{4^{l}} .
$$

Neglecting the energy dissipated by friction and imperfect elasticity, we have

$$
\begin{gathered}
T+U^{\prime}+U^{\prime \prime}=\text { const. } \\
\therefore \quad \frac{m a \dot{y}_{a}^{2}}{4}+\frac{s^{2} \pi^{2} F}{\mathrm{I} \sigma a} y_{a}^{2}+\frac{m g a}{4 l} y_{a}{ }^{2}=\text { const. }
\end{gathered}
$$


By differentiating the energy equation and simplifying we obtain

$$
\ddot{y}_{a}+\left(\frac{g}{l}+\frac{s^{2} \pi^{2} F}{4 m \alpha^{2}}\right) y_{a}^{\prime}=0 .
$$

From (Io) we have for the frequency equation

$$
N_{s}=\frac{I}{2 \pi} \sqrt{\frac{g}{l}+\frac{s^{2} \pi^{2} F}{4 m a^{2}}} .
$$

If $s$ be given the values $\mathrm{I}, 3,5,7, \ldots$ or the values $2,4,6,8, \cdots$, the values of $N_{s}$ do not vary in the same or any multiple ratio. Hence the frequencies of a horizontally suspended coiled spring do not form a harmonic series.

If in equation (I I) $g / l$ be put equal to zero we shall have the frequency equation for a coiled spring uninfluenced by gravity; if the other term under the radical be put equal to zero we have the frequency of the gravity pendulum; calling the latter $N_{0}$ and the former $N_{s}^{\prime}$, (I I) may be written in the following form:

$$
N_{s}=\sqrt{N_{0}^{2}+N_{s}^{\prime 2}}
$$

The frequency equation ( I I) may be verified by determining frequencies experimentally and by computing them from the constants of the spring: Instead of doing this I have adopted the following method: If $N_{0}$ represents the pendulum frequency of the suspended spring ( I I) may be written as follows:

$$
N_{s}=\sqrt{N_{0}^{2}+s^{2} k}
$$

in which $k$ is a constant. Giving some particular value, say 2, we have

$$
\begin{gathered}
k=\frac{N_{2}^{2}-N_{0}^{2}}{4} \\
\therefore N_{s}=\sqrt{N_{0}^{2}+\frac{s^{2}}{4}\left(N_{2}^{2}-N_{0}^{2}\right) .}
\end{gathered}
$$

If the two frequencies $N_{0}$ and $N_{2}$ be determined experimentally all the other frequencies can be computed by substitution in equation( I2). This was done for all frequencies up to $N_{10}$, and the results compared with experimental values. 
Frequencies of Horizontal Coiled Spring.

\begin{tabular}{c|c|c||c}
\hline$N_{s}$ & Observed. & Computed. & $N_{\text {' Computed. }}$ \\
\hline$N_{0}$ & .697 & - & .000 \\
$N_{1}$ & .785 & .790 & .372 \\
$N_{2}$ & 1.020 & - & .744 \\
$N_{3}$ & 1.31 & 1.31 & 1.116 \\
$N_{4}$ & 1.63 & 1.63 & 1.49 \\
$N_{5}$ & 1.99 & 1.98 & 1.85 \\
$N_{6}$ & 2.35 & 2.33 & 2.23 \\
$N_{7}$ & 2.69 & 2.67 & 2.60 \\
$N_{8}$ & 3.08 & 3.05 & 2.98 \\
$N_{9}$ & 3.42 & 3.42 & 3.35 \\
$N_{10}$ & 3.77 & 3.78 & 3.72 \\
\hline
\end{tabular}

In the last column of the above table are given, for the sake of comparison, the frequencies which the spring would have had if the vibration were wholly due to elasticity. As was to be expected the actual frequencies of the higher components are not much influenced by the pendulum suspension. This is not the case, however, with the lower components.

The differences between the computed and observed frequencies are well within the errors of observation. The latter were probably in the neighborhood of a quarter per cent. Considering the crudeness of the spring and the assumptions made in the mathematical demonstration it was not thought worth while to strive for greater accuracy in the experimental verification. Besides the assumptions explicitly mentioned, the use of the calculus implicitly assumes that the spring is continuous like a rod or tube instead of being made up of widely separated spires, also that the method of suspension is continuous instead of being limited to a few widely separated threads.

STANFORD UnIVERSITY,

May, 1902. 